\title{
Control Algorithms for Large-scale Single-axis Photovoltaic Trackers
}

\author{
Dorian Schneider \\ Institute of Imaging \& Computer Vision, RWTH Aachen University, 52056 Aachen \\ Corresponding author: schneider@lfb.rwth-aachen.de
}

\begin{abstract}
The electrical yield of large-scale photovoltaic power plants can be greatly improved by employing solar trackers. While fixed-tilt superstructures are stationary and immobile, trackers move the PV-module plane in order to optimize its alignment to the sun. This paper introduces control algorithms for single-axis trackers (SAT), including a discussion for optimal alignment and backtracking. The results are used to simulate and compare the electrical yield of fixed-tilt and SAT systems. The proposed algorithms have been field tested, and are in operation in solar parks worldwide.
\end{abstract}

Keywords: single-axis solar tracker, backtracking, photovoltaic, sun tracking.

\section{Introduction}

The degree of efficiency of photovoltaic (PV) power plants can be maximized by optimizing the alignment of the photovoltaic module plane to the current position of the sun. Unlike fixed-tilt superstructures, where modules are placed stationary in the field, tracking superstructures mount the modules on carriers able to rotate along one or two axes in order to maximize the electrical yield of the system. Two different schemes for the construction of a PVtracker are in existence: Dual-Axis Trackers (DAT) are provided with two degrees of freedom, which theoretically allows optimal module-sun alignment and hence the maximum possible yield at any time. Theoretically, DATs enable a rise of 30-45\% [7] in yield compared to an optimally-positioned fixed-tilt plant at the same location. The details for tracker performances and extra yield have high variance, since they depend strongly on the location where the system is installed [4]. The benefits of DATs are achieved at the cost of a need for more space and at the cost of higher mechanical complexity, leading to higher costs for construction, planning and maintainance. The second scheme, Single-Axis Trackers (SAT), provides only one degree of freedom, limiting the tracking motion so that perfect module-sun alignment cannot always be provided.

Although the tracking angle is limited, the system can provide incremented yields of 10-20\% compared to a perfectly adjusted fixed-tilt plant at the same location. The constructional complexity is significantly lower than for DATs, which has a positive effect on the costs for construction and maintainance.

In order to choose the best system for a power plant of given size and location, it is necessary to optimize the cost-yield ratio of the system. While the costs for construction and maintainance can be estimated easily, expected yields can only be analysed using sophisticated simulations.

While several algorithms and control schemes for high precision DAT control have been published $[1,2,8,9]$, very few publications have handled the control of SATs. In fact, the results found in $[5,6]$ are estimations, and give not an exact solution for the backtracking problem. For this reason, our paper introduces control algorithms for SATs which allow the system to be regulated to the optimal position at any time, without the need for any additional sensor technology or hardware, but using the calculated sun position instead.

The problem of self-shadowing is addressed with a high-precision, field-tested backtracking algorithm. The results are used for a basic energy yield analysis.

The structure of the paper is as follows. In Section 2, the nomenclature and coordinate system for later sections are defined. Section 3 handles the basic mathematical equations for naive tracking, allowing the system to find the rotation angle that maximizes the yield for a given sun position. Since the system should avoid self-shadowing by all means, a backtracking algorithm that finds the optimal shadow free rotation angle is discussed in Section 4. The results are affiliated in Section 5 to simulate SAT yield expectations for different latitudes and superstructure settings in Matlab/Simulink. Section 6 discusses the results, and Section 7 concludes the work.

\section{Coordinate system}

We define the nomenclature and coordinate system that is used throughout the subsequent sections. Due to the rotational movement of the sun, a polar coordinate system is used, as illustrated in Fig. 1: Here, the azimuth angle $\phi$ is given by the angle between the orthogonal projection of the sun vector $s$ to the 

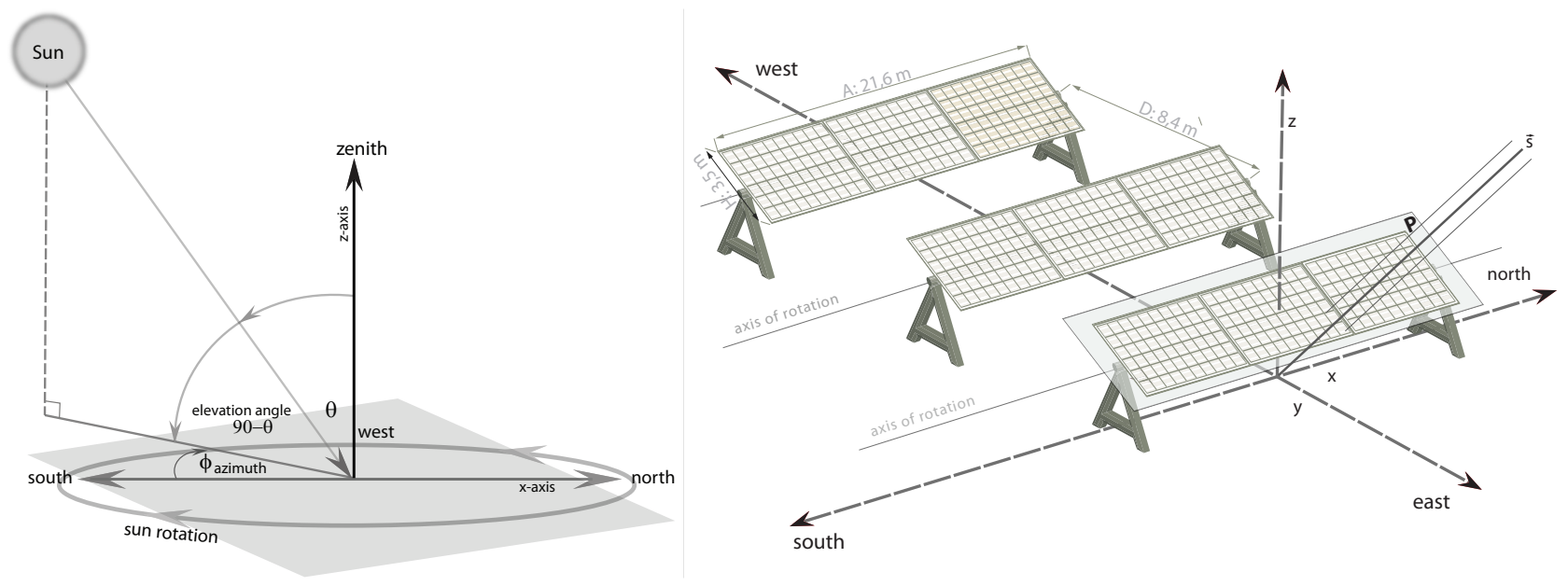

Figure 1: Schemes of the SAT coordinate system. Left: illustration of the sun azimuth and elevation angles. Right: Illustration of three adjacent SATs with corresponding distances and annotations.

$x y$-plane and the $x$-axis (north-south axis). The azimuth takes positive values $\left[+180^{\circ}, 0^{\circ}\right.$ [ when the sun is eastward, and negative values $\left.] 0^{\circ},-180^{\circ}\right]$ when it is westward. It is zero when the sun is in the south. The zenith angle $\theta$ gives an indication for the sun altitude by measuring the angle between the orthogonal projection of the sun vector $s$ to the $x y$-plane and the $z$-axis. The sun elevation $\theta_{e}$ angle is then given by $90^{\circ}-\theta$.

An optimal SAT alignment would be lengthwise, parallel to the $x$-axis of the coordinate system, i.e. perpendicular to the east-west axis. If the environmental setting of the power plant prohibits optimal alignment of the SAT superstructure, a deviation angle $\eta$ occurs, as illustrated in Fig. 2. As nomenclature we define $\eta<0$ for clockwise rotation and $\eta>0$ for counter-clockwise rotation. For simplicity, we do not rotate the SAT system, but we rotate the sun azimuth instead. The new sun azimuth becomes

$$
\phi_{\eta}=\phi-\eta
$$

One can easily convert between polar and Cartesian coordinates using:

$$
\begin{aligned}
& x_{s}=r \cos \phi_{\eta} \sin \theta_{e}, \\
& y_{s}=r \sin \phi_{\eta} \sin \theta e, \\
& z_{s}=r \cos \theta_{e} .
\end{aligned}
$$

\subsection{Sun path mirroring}

The sun starts its course early in the day in the east, reaches the south around noon, and ends its path in the evening in the west. The course of the sun is always symmetric with regard to the north-south axis. This fact is exploited to further simplify the subsequent calculations: A variable $\delta$ is introduced, according to:

$$
\delta= \begin{cases}+1 & \text { sun is in the east, i.e. } \left.\left.\psi_{\eta} \in\right] 0^{\circ}, 180^{\circ}\right], \\ -1 & \text { sun is in the west, i.e. } \left.\left.\psi_{\eta} \in\right]-180^{\circ}, 0^{\circ}\right] .\end{cases}
$$

Using $\delta$, the system behaviour must only be calculated once during simulation for either west or east. The results are then mirrored to get the final result.

\subsection{Plane inclination}

Solar power plants are sometimes installed on nonplane surfaces - for example in a hilly environment. The inclination of the surface must be taken into account for precise tracking. The scheme for a nonplane setting is illustrated in Fig. 3. To keep all calculations as simple as possible, the plane inclination is embedded into the SAT rotation angle by following the update rule:

$$
\alpha=\alpha-\delta \cdot \beta \text {. }
$$

\section{$3 \quad$ Basic tracking}

According to Lambert's law, the yield of a solar module is directly proportional to the angle of incidence $\gamma$ between the sun light and the module normal vector for a given insolation intensity. The lower the angle, the higher the irradiation intensity, and the higher the yield. Since the loss in irradiation intensity follows the function $\cos \gamma$, maximal yield can only be achieved when the sun vector is orthogonal to the module plane, i.e. $\gamma=0$. A single-axis tracker (unlike a DAT) cannot always achieve orthogonality, due to the mechanical restrictions that apply, but the yield can be optimized during the span of one day. This section aims to express the system rotation angle $\alpha$ as a function of the sun position in order to achieve maximum yield. 


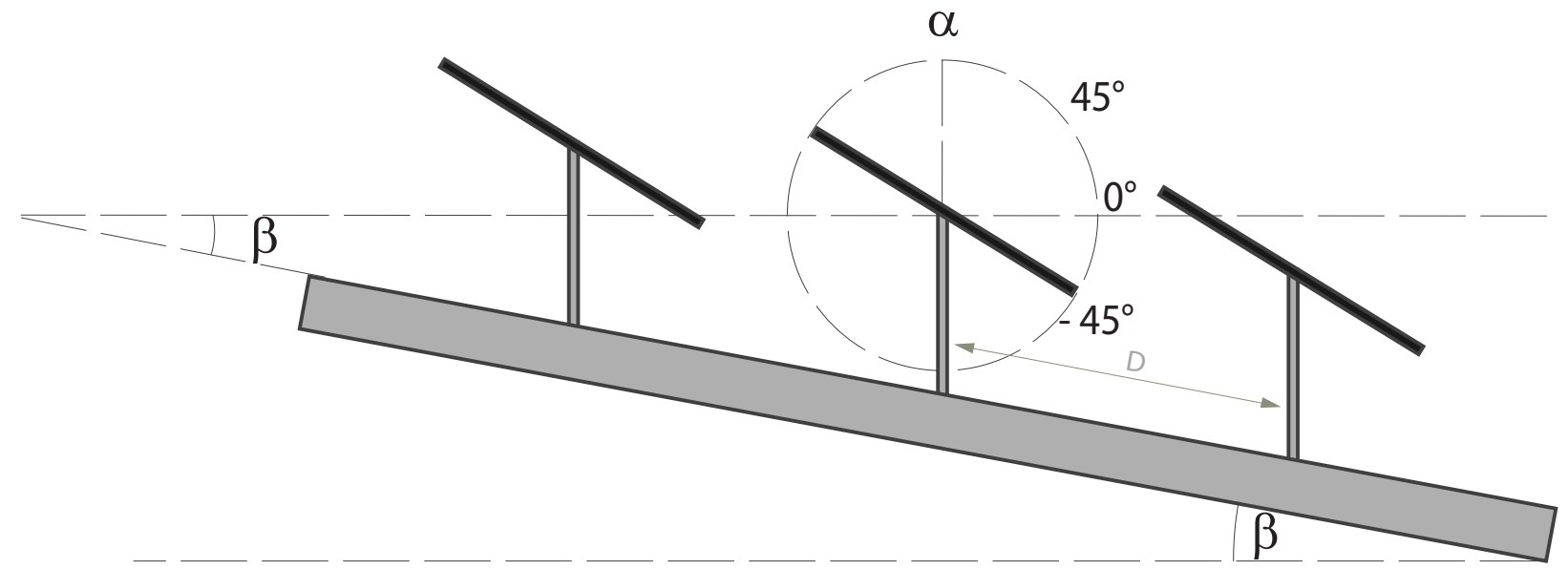

Figure 3: Illustration of the plane inclination angle $\beta$ for hilly environments.

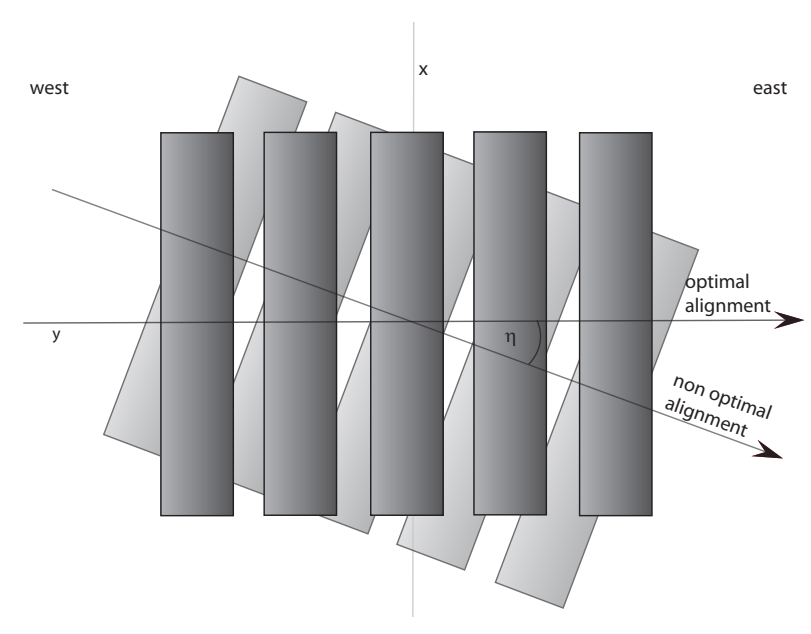

Figure 2: Explanatory scheme for the SAT misalignment angle $\eta$. The system is not optimally aligned on the east-west axis ( $y$-axis) for $\eta \neq 0$.

Let the module plane of one SAT be denoted as $\boldsymbol{P}$, cf. Fig. 1 b. $\boldsymbol{P}$ can be rotated around the $x$-axis with the rotation angle $\alpha$, but has no other degrees of freedom. In this way, $\boldsymbol{P}$ can be expressed in matrix notation:

$$
\boldsymbol{P}=\left[\begin{array}{cc}
1 & 0 \\
0 & \cos \alpha \\
0 & \sin \alpha
\end{array}\right] .
$$

The angle $\alpha$ is negative when $\boldsymbol{P}$ is inclined eastward, positive when $\boldsymbol{P}$ is inclined westward. The origin of co-ordinates $\boldsymbol{O}=[0,0,0]^{\top}$ is placed as indicated in Fig. 4. A normal vector $\boldsymbol{v}$ of $\boldsymbol{P}$ can be expressed by:

$$
\boldsymbol{v}=\operatorname{det} \boldsymbol{P}=\left|\begin{array}{cc}
1 & 0 \\
0 & \cos \alpha \\
0 & \sin \alpha
\end{array}\right|=\left[\begin{array}{c}
0 \\
-\sin \alpha \\
\cos \alpha
\end{array}\right] \text {. }
$$

As mentioned above, the maximal yield is equivalent to the minimal angle $\gamma$ between the normal vector $\boldsymbol{v}$ and the sun vector $\boldsymbol{s}$. This can be achieved by minimizing the absolute value of the cross product $\boldsymbol{n}$ between the two vectors:

$$
\begin{aligned}
\boldsymbol{n} & =\boldsymbol{v} \times \boldsymbol{s}=\left[\begin{array}{c}
0 \\
-\sin \alpha \\
\cos \alpha
\end{array}\right] \times\left[\begin{array}{l}
x \\
y \\
z
\end{array}\right] \\
& =\left[\begin{array}{c}
y \cos \alpha+z \sin \alpha \\
x \cos \alpha \\
x \sin \alpha
\end{array}\right] .
\end{aligned}
$$

Minimizing the absolute value of (8) corresponds to minimizing the area of the parallelogram that the two vectors span, and hence corresponds to minimizing the angle between them:

$$
\begin{aligned}
|\boldsymbol{n}|^{2} & =(y \cos \alpha+z \sin \alpha)^{2}+(x \cos \alpha)^{2}+(x \sin \alpha)^{2} \\
& =(y \cos \alpha+z \sin \alpha)^{2}+x^{2} .
\end{aligned}
$$

From (9) it can be seen that perfect SAT alignment (i.e. $|n|^{2}=0$ ) can only be achieved when the sun is positioned on the east-west axis $\left(x^{2}=0\right)$. The best SAT rotation angle for a given sun position can be found when

$$
0=y \cos \alpha+z \sin \alpha
$$

applies. Solving for $\alpha$ and applying (5) gives

$$
\alpha=-\arctan \frac{y}{z}-\delta \cdot \beta
$$

as the optimal rotation angle. Care needs to be taken when the sun is rising or setting, i.e. $z=0$.

\section{Backtracking}

Equation (11) allows the system to find an optimal rotation angle for a given sun position at any time. 


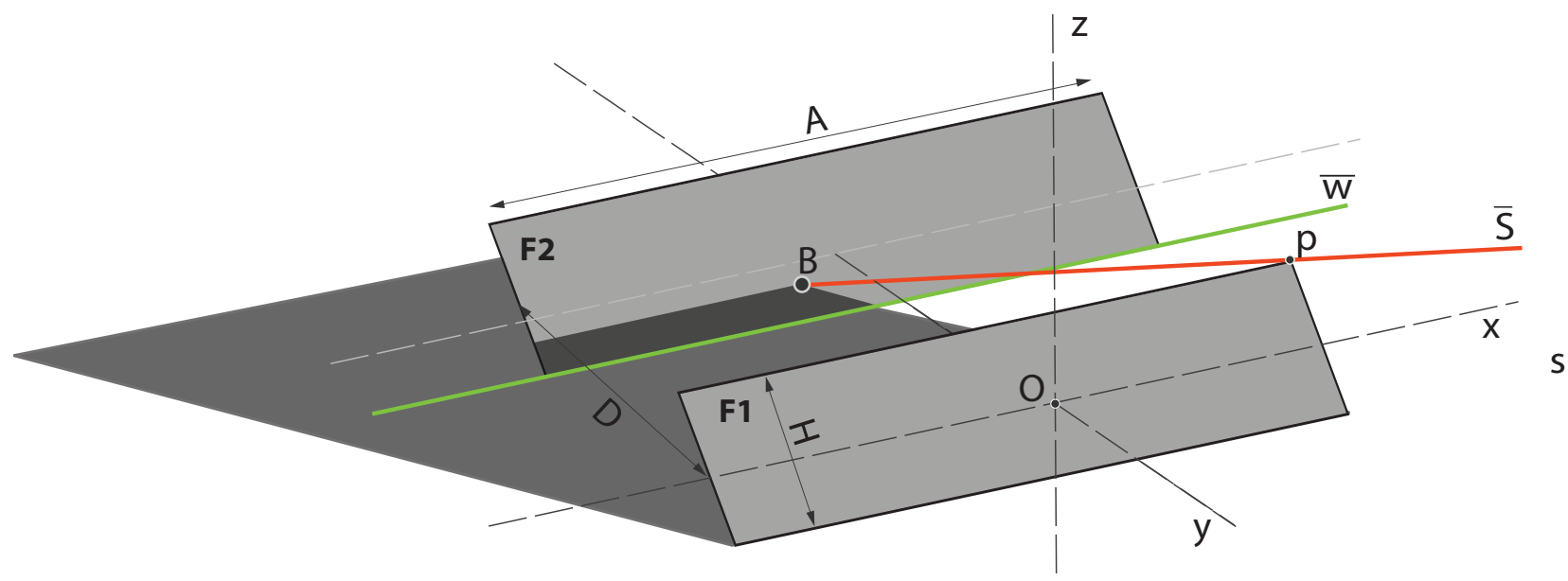

Figure 4: Illustration of planes, lines and points needed for deriving the backtracking algorithm: A shadow is cast from surface $F_{1}$ to surface $F_{2}$. By rotating SAT so that line $s$ and line $\boldsymbol{w}$ intersect, shading can be avoided by guaranteeing a minimal deviationfrom the optimal rotation angle.

However, the case is not considered when, for specific sun positions, self-shading may occur between adjacent SATs. The problem is illustrated for a twounit SAT in Fig. 4. With more units, the problem becomes even more important. In order to maximize the yield, shading should be avoided by all means, even though a shadow-free position is not optimal in terms of the module-sun alignment, as discussed in Section 3. Smart control algorithms should be able to detect when SAT self-shading occurs and hence update the current rotation angle so that no shading can occur, while still optimizing the module alignment for maximum yield. This procedure is known as backtracking. The next section derives the equations for a shadow-free SAT backtracking control mechanism that maximizes the electrical yield.

First, it is necessary to check whether self-shading occurs for a given sun vector $\boldsymbol{s}$. As illustrated in Fig. 4, the module surface of two adjacent SATs of width $A$ and height $H$ will be denoted as $F_{1}$ and $F_{2}$, respectively. The origin of the co-coordinates is placed at the center of gravity of $F_{1}$. In order to calculate whether a shadow is cast from $F_{1}$ to $F_{2}$, one may project the corner point $\boldsymbol{p}$ of surface $F_{1}$ onto surface $F_{2}$ along the sun vector $\boldsymbol{s}$. Point $\boldsymbol{p}$ can be expressed in Cartesian coordinates as:

$$
\begin{aligned}
\boldsymbol{p} & =\left[\begin{array}{lll}
\frac{A}{2} & y_{p} & z_{p}
\end{array}\right]^{\top}, \\
\text { with } \quad y_{p} & =\frac{D}{2} \cos \alpha \\
\text { and } \quad z_{p} & =\frac{D}{2} \sin \alpha .
\end{aligned}
$$

The line $\overline{\boldsymbol{s}}$ that passes through $\boldsymbol{p}$ and is parallel to the sun vector $s$ can be expressed as:

$$
\bar{s}: p+\lambda s
$$

According to equation (7), the normal vector $\boldsymbol{v}$ of surface $F_{1}$ corresponds to:

$$
\boldsymbol{v}=\left[\begin{array}{c}
0 \\
-\sin \alpha \\
\cos \alpha
\end{array}\right] \text {. }
$$

The plane that surface $F_{2}$ is part of can be parametrized by

$$
F_{2, \text { plane }}: \boldsymbol{v} \cdot \boldsymbol{r}-b=0,
$$

where $\boldsymbol{r}$ is an arbitrary point on the plane and $b$ is a constant that must be determined. We choose $r=$ $\left[\begin{array}{lll}0 & D & 0\end{array}\right]^{\top}$ and rearrange for $b$ to find

$$
b=-D \sin \alpha .
$$

Insertion of (12) for vector $\boldsymbol{r}$ into (14) and rearranging for $\lambda$ gives:

$$
\begin{aligned}
& \boldsymbol{v} \cdot(\boldsymbol{p}+\lambda \boldsymbol{s})-b=0, \\
\text { thus } \quad & \lambda=\frac{b-\boldsymbol{v} \cdot \boldsymbol{p}}{\boldsymbol{v} \cdot \boldsymbol{s}}=\frac{b}{\boldsymbol{v s}}=-\frac{D \sin \alpha}{z_{s} \cos \alpha-y_{s} \sin \alpha} .
\end{aligned}
$$

We are now in a position to detect whether shading from surface $F_{1}$ onto $F_{2}$ occurs: By inserting (16) into (12), one finds the Cartesian coordinates of the planeline intersection point $\boldsymbol{B}$ (cf. Fig. 4). Since $\boldsymbol{B}$ can lie anywhere on the plane and must not necessarily lie on surface $F_{2}$, an interval check must be performed to test whether $\boldsymbol{B}$ is inside the bounding box of $F_{2}$. If the check is positive, shading occurs and a new, shadow-free rotation angle should be found.

If shading occurs, the tracker should be rotated as far as the lower bound of surface $F_{2}$ (green line in 


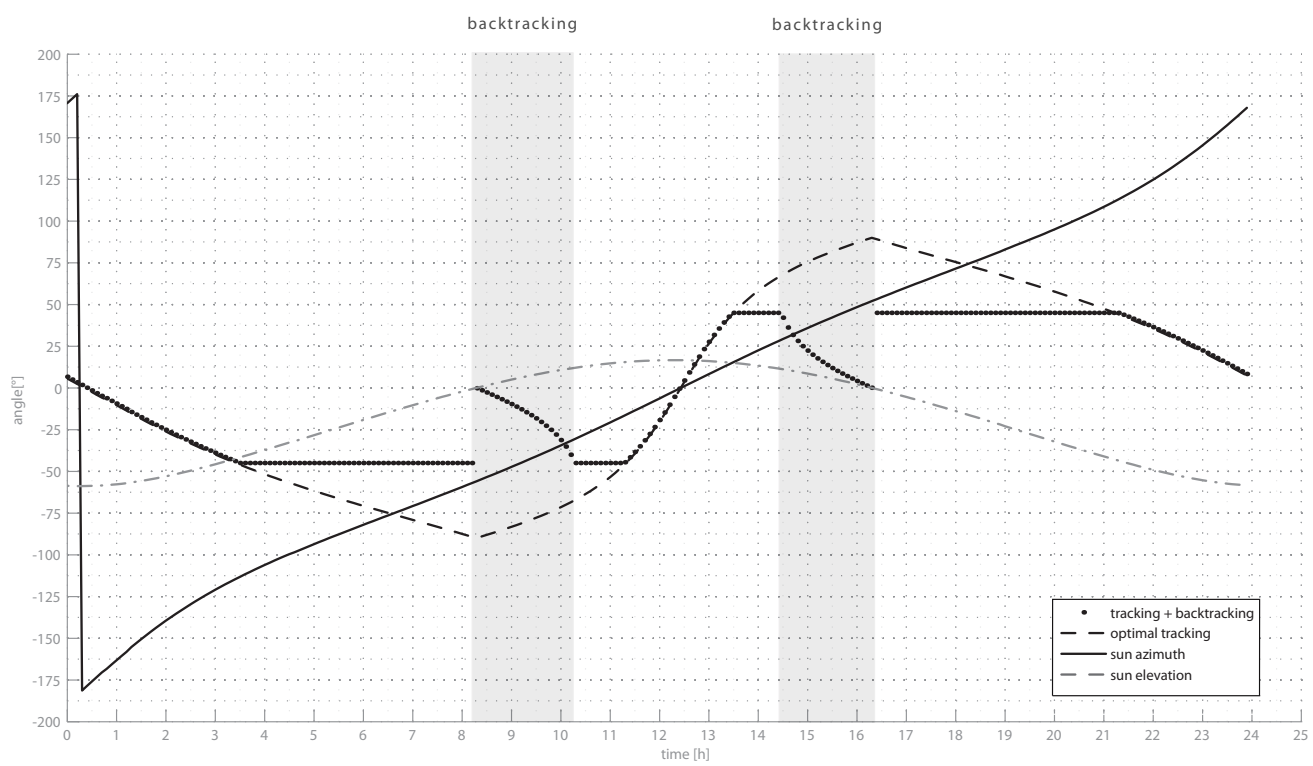

Figure 5: Tracking and backtracking angles, sun azimuth and elevation plotted for January 1st, 2012 in Berlin. The maximal rotation angle has been clipped to $\pm 45^{\circ}$. As the sun elevation angle is low, backtracking occurs from 8 am to $10 \mathrm{am}$, and also from $14.30 \mathrm{pm}$ and $16.30 \mathrm{pm}$.

Fig. 4 denoted as $\overline{\boldsymbol{w}}$ ) intersects with line $\overline{\boldsymbol{s}}$. Line $\overline{\boldsymbol{w}}$ can be parametrized by

$$
\overline{\boldsymbol{w}}:\left[\begin{array}{c}
-\frac{A}{2}+\mu A \\
-y_{p}+D \\
-z_{p}
\end{array}\right]
$$

To find the rotation angle that avoids shading, the intersection point between $\overline{\boldsymbol{s}}$ and $\overline{\boldsymbol{w}}$ must be found by equalizing the line expressions $\overline{\boldsymbol{w}}=\overline{\boldsymbol{s}}$, thus

$$
\left[\begin{array}{c}
-\frac{A}{2}+\mu A \\
-\frac{D}{2} \cos \alpha+D \\
-\frac{D}{2} \sin \alpha
\end{array}\right]=\left[\begin{array}{c}
-\frac{A}{2}+\lambda x_{s} \\
\frac{D}{2} \cos \alpha+\lambda y_{s} \\
\frac{D}{2} \sin \alpha+\lambda z_{s}
\end{array}\right] .
$$

This gives us a nonlinear system with three unknowns $(\alpha, \lambda, \mu)$ and three equations that we solve numerically to find two solutions for the shadow-free rotation angle (including (5)) $\alpha_{\mathrm{sf}}$ :

$$
\begin{aligned}
\alpha_{\mathrm{sf}, 1} & =\arccos \frac{a-\sqrt{b}}{c}-\delta \beta, \\
\alpha_{\mathrm{sf}, 1} & =\arccos \frac{a+\sqrt{b}}{c}-\delta \beta,
\end{aligned}
$$

where $\quad a=H D z_{s}^{2}$,

$$
\begin{aligned}
& b=H^{4} y_{s}^{4}+H^{4} y_{s}^{2} z_{s}^{2}-H^{2} D^{2} y_{s}^{2} z_{s}^{2}, \\
& c=H^{2} y_{s}^{2}+H^{2} z_{s}^{2} .
\end{aligned}
$$

The actual values for $\lambda$ and $\mu$ are of no interest for our problem. It is intuitive that the system angle $\alpha_{\text {sf }}$ should be minimal. Obviously, equation (19) does not meet this requirement, which leaves us with

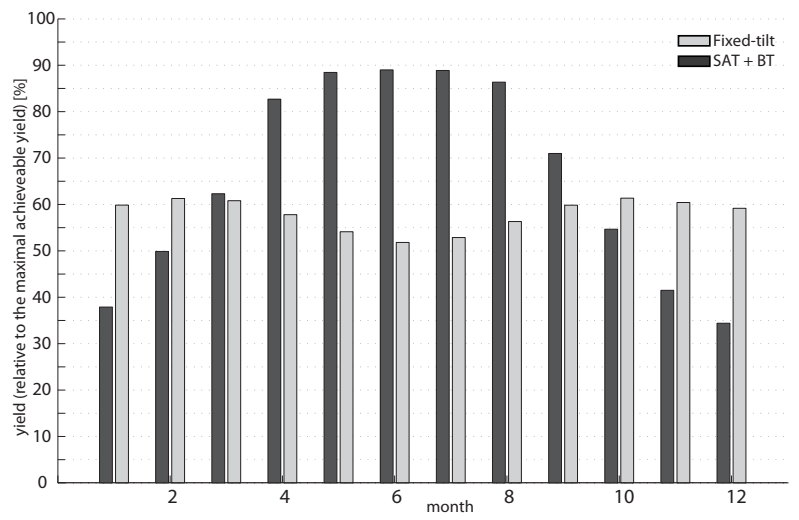

Figure 6: Comparison of fixed-tilt yields and SAT yields for each month of a year. The results are normed to the maximum yield that could be achieved with a perfectly aligned module plane.

the final solution (20) for the best, shadow-free backtracking angle.

The proposed backtracking algorithm has been tested in the Arizona desert with a real SAT system. The algorithmic framework proved to be exact at centimetre precision. Meanwhile, more than $100 \mathrm{MW}$ of SATs worldwide are controlled using the proposed scheme.

\section{Results}

Equations (20) and (11) allow us to find an optimal, shadow-free operation angle for SAT. However, the exact position of the sun is needed for a given time and location. For this purpose, our paper uses the 


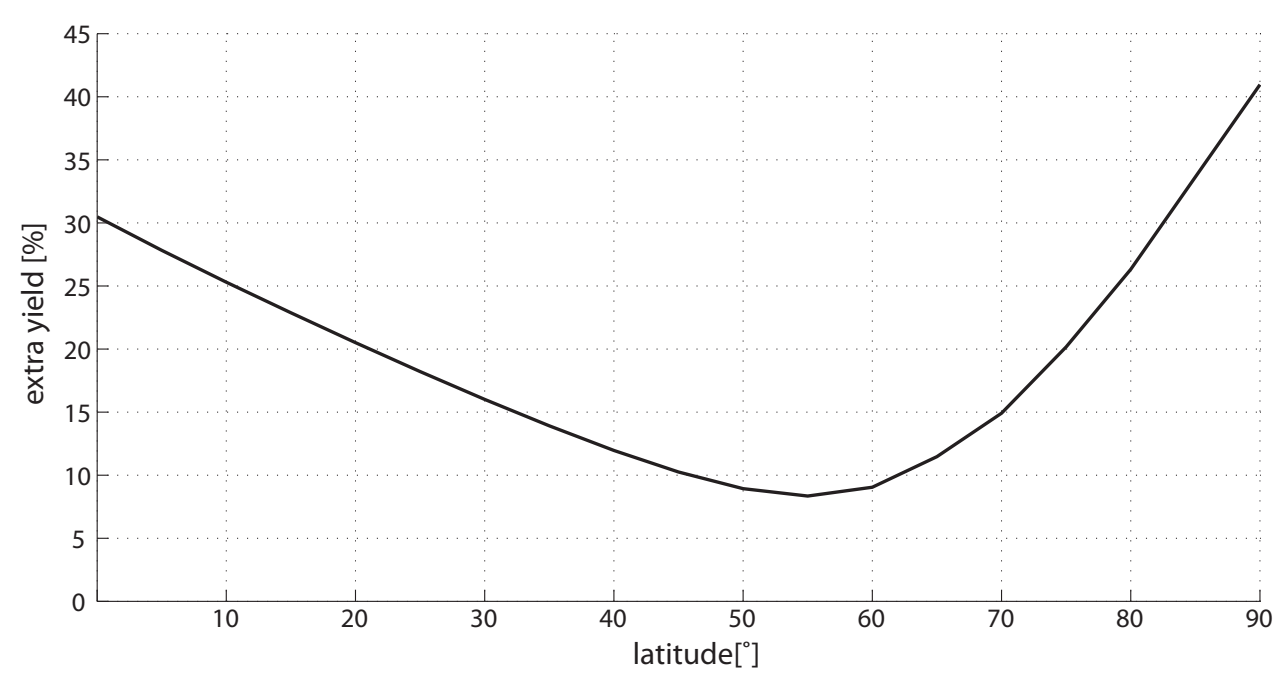

Figure 7: Averaged, annual extra yield of SAT compared to an optimally aligned fixed-tilt superstructure in dependence on latitude.

sun position algorithm proposed in [10], which allows us to determine the sun azimuth and elevation with accuracy of $\pm 0.0003^{\circ}$, without the need for any additional sensors or hardware. In fact, the sun position algorithm can run on the same controller as the tracking control, which is beneficial in terms of costs and installation complexity. Fig. 5 shows a simulation of the SAT behaviour during one day in January for the location in Berlin, Germany. The shadow-free angle of operation $\alpha_{\mathrm{sf}}$ and the optimal angle $\alpha$ are plotted against time. Additionally, the sun azimuth and elevation are shown. The angle $\alpha_{\text {sf }}$ is limited to a range of $\left[-45^{\circ}, 45^{\circ}\right]$, due to mechanical limitations that apply to real-world structures. As a matter of fact, all SATs are provided with a rotation limit, which may vary between $45^{\circ}$ and $60^{\circ}$, depending on the design. The system switches to backtracking as soon as the sun rises, and continues shadow-free tracking until about $10 \mathrm{am}$. Afterwards there is no longer any shading, and the system's angle of operation corresponds to the optimal angle until about $2.30 \mathrm{pm}$, when the sun elevation has declined enough to cause shading again.

The results were further used to simulate the relative extra yield of SAT compared to an optimally aligned fixed-tilt superstructure at the same location, cf. Fig. 6. The results shown here are normed to the maximal achievable yield. It can be seen that SAT clearly outperforms the fixed-tilt installation in the summer months, when the sun stands high in the sky and the days are long. In the winter months, the fixed-tilt installation performs better because the sun stands low in the sky, shadows are more likely and the SAT system often switches to backtracking, hence missing the optimal alignment to the sun. However, the results are relative to the maximal achievable yield and have no information about the absolute an- nual extra yield. Fig. 7 shows the simulation results for the total, absolute extra yield of SAT compared to an optimally aligned fixed-tilt installation. The plot shows the extra yield plotted against the latitude. The clear-sky insolation model proposed in [3] has been used for this purpose. It is interesting to see how minimal gain can be achieved for latitudes of around $50^{\circ}$ to $60^{\circ}$, which correspond to central Europe. Here, the simulation predicts an extra yield of about $8-10 \%$, which is consistent with the results found by other researchers [11]. According to Fig. 7, the usage of a SAT system becomes very lucrative for southern countries, where the gain may rise up to $30 \%$.

Finally, the effect of the spacing $D$ between two adjacent SATs has been investigated in this work. Fig. 8 summarizes the results. It can be seen that in general the best distance in terms of extra gain and space efficiency can be achieved for a distance of about 12 meters, when the slope of the curve flattens out. However the best distance depends strongly on the park layout, and generally needs to be selected individually for each park. When dealing with diffuse insolation (which forms a major part of central Europe's insolation), a totally flat tracker would be the best operational position. Theoretically, the maximum amount of light could be collected in this position. However, to detect the current type of insolation, extra sensors are required. Alternative solutions could employ local weather broadcasts to adapt the tracking behaviour in order to avoid external hardware.

\section{Conclusions}

We have proposed an algorithmic framework for controlling photovoltaic single-axis trackers using back- 


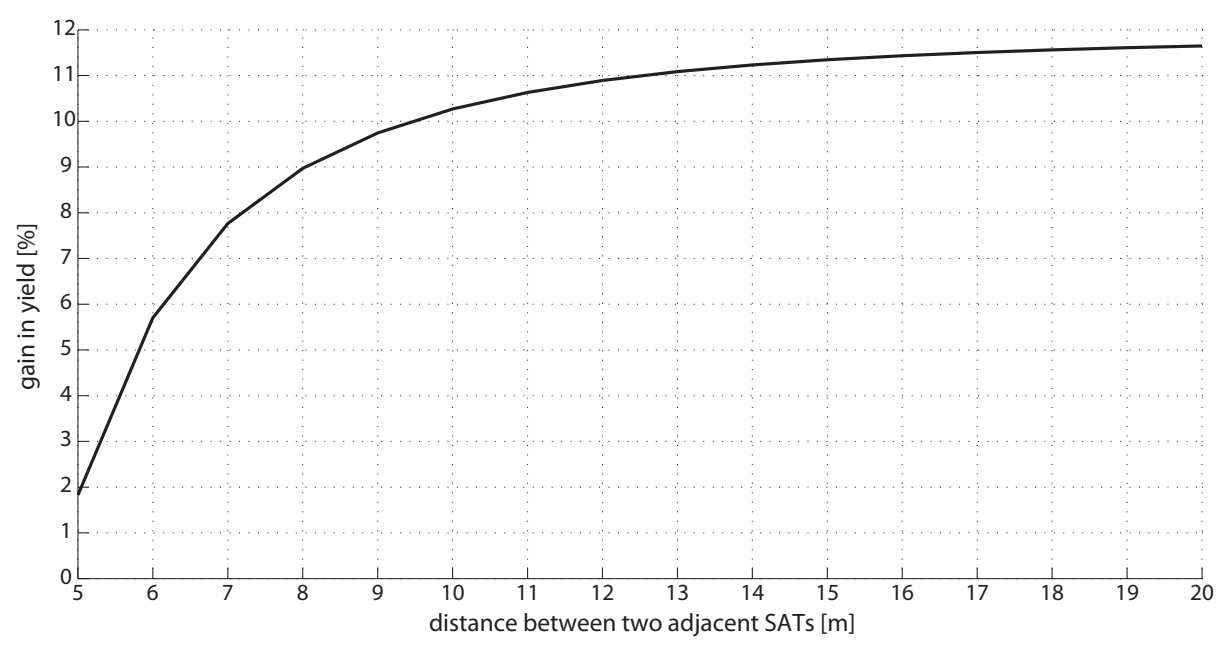

Figure 8: Averaged, annual extra yield of a SAT compared to an optimally aligned fixed-tilt superstructure in dependence on the distance $D$ between two adjacent SAT units.

tracking. The control schemes are able to maximize the electrical yield of a SAT power plant by finding the best, i.e. shadow-free, rotation angle for the tracker in dependence on its location and environmental settings. In the simulation, the SAT yields have been compared to fixed-tilt yields, giving an insight into the strengths and weaknesses of the system. The proposed control scheme has been field tested, and is in use in power plants with more than $100 \mathrm{MW}$ output worldwide.

\section{References}

[1] A. Catarius, M. Christiner. Azimuth-altitude dual axis solar tracker. Bachelor thesis, Worcester Polytechnic Institute, 2010.

[2] T. N. Kathiba, A. Mohamed, R. J. Khan et al. A Novel Sun Tracking Controller For Photovoltaic Panels. Journal of Applied Sciences 9(22):4050 4055, 2009.

[3] B. Keller, A. M. S. Costa, A Matlab GUI for calculating the solar radiation and shading of surfaces on the earth. Computer Applications in Engineering Education 19(1):161-170, 2009.

[4] D. L. King, W. E. Boyson, J. A. Kratochvil. Analysis of Factors Influencing the Annual Energy Production of Photovoltaic Systems. In Photovoltaic Specialists Conference, 2003

[5] E. Lorenzo, L. Navarte, J. Muoz. Tracking and back-tracking. Progress in Photovoltaics: Research and Applications 19:747-753, 2011.

[6] E. Lorenzo, M. Perez, A. Ezpeleta et al. Design of tracking photovoltaic systems with a single vertical axis. Progress in Photovoltaics: Research and Applications 10: 533-543, 2002.
[7] T. M. Pavlovic, D. D., Milosavljevic, A. R. Radivojevic et al. A comparison and assesment of electricity generation capacity for different types of photovoltaic solar plants of $1 \mathrm{MW}$ in Sokobanja, Serbia. Thermal Science 15:605$618,2011$.

[8] T. Peterson, J. Rice, J. Valentin. Solar tracker. Final project report, Cornell University, 2005.

[9] S. Seme, G. Stumberger, J. Vorsic. Maximum efficiency trajectories of a two-axis sun tracking system determined considering tracking system consumption. IEEE Transactions on Power Electronics 26:1280-1290, 2011.

[10] I. Reda, A Andreas. Solar Position Algorithm for Solar Radiation Applications Solar Energy $7(5): 577-589,2004$.

[11] P. Vanicek, S. Stein. Simulation of the impact of diffuse shading on the yields of large single-axis tracked PV Plants. Technical Report, Deutsche Gesellschaft fuer Sonnenenergie LV Berlin Brandenburg e.V., 2009. 